\title{
Sobre la red asistencial de la salud mental de niños y adolescentes
}

Quisiéramos hacer, de nuevo, algunas consideraciones sobre la situación de la atención a la salud mental de niños y adolescentes. En primer lugar señalar que el debate planteado en los medios de comunicación, a raíz de la denuncia de unos padres por el ingreso de su hijo de 9 años en una unidad hospitalaria de adultos, no es de ninguna manera una situación nueva. Tanto desde nuestra Asociación, como desde diferentes colectivos de profesionales, llevamos al menos cinco años señalando la carencia y precariedad de los recursos asistenciales para niños y adolescentes y la necesidad de desarrollar y completar la red asistencial.

En 1995 la Asociación Madrileña de S.M. elaboró un informe sobre los programas de atención ambulatoria en la $\mathrm{Co}$ munidad de Madrid, en el que se señalaba la falta de desarrollo de los programas. Mas adelante en 1998, la Sección Infanto-Juvenil de la AEN realizó un informe sobre la atención a niños y adolescentes en las diferentes Comunidades Autónomas, en el que quedaba patente que la carencia de recursos era una situación general en todo el Estado. En este mismo año un grupo de expertos, por encargo del Servicio Regional de Salud de la Comunidad de Madrid, realizó un informe sobre la situación asistencial, analizando los programas ambulatorios, recursos hospitalarios y dispositivos intermedios, así como la previsión de necesidades.

Desde entonces podemos asegurar que la situación sigue siendo cualitativamente la misma. La red de atención solo esta implantada a nivel de programas ambulatorios y todavía de manera insuficiente. Puede ser que haya aumentado algún psiquiatra y/o psicólogo (también es cierto que alguna plaza se ha reconvertido para la atención a adultos), puede haber algún dispositivo más (un hospital de día concertado, para niños, en Madrid). Pero estructuralmente los programas de atención ambulatoria no están presentes ni en todas las áreas, ni en todas las Comunidades Autónomas. A esto hay que añadir que el numero de profesionales clínicos (psiquiatras y psicólogos) es insuficiente y todavía en muchos equipos siguen atendiendo simultáneamente adultos y niños, principalmente en Madrid. Esta situación es insostenible principalmente por dos razones. Una porque el tiempo real que se dedica a los programas infanto-juveniles es mínimo. Dos porque los profesionales de adultos no están especializados en la atención a niños y adolescentes.

En cuanto a las unidades de ingreso hospitalario para adolescentes podemos 
seguir afirmando que son prácticamente inexistentes. Nos referimos a unidades especificas. Especificidad que viene dada por las características de la psicopatologia del adolescente, tan diferente a la de los adultos. Pacientes entre los 13 y los 17 años, que precisarían una unidad de ingreso en hospitales generales (nunca un centro psiquiátrico monográfico, modelo obsoleto y afortunadamente abandonado) con unos objetivos y un tratamiento terapéutico, que naturalmente no tiene nada que ver con el que se realiza en las unidades de adultos. Este programa debe ser realizado por un equipo interdisciplinario (psiquiatras, psicólogos, enfermeros, educadores, etc.) especializado en la atención a niños y adolescentes. Por lo tanto el problema no se resuelve ingresando a los menores en habitaciones individuales, en las unidades de adultos.

En cuanto a los niños menores de 13 años, el ingreso debería realizarse en los Servicios de Pediatría. Pero sabemos que en la practica esto es imposible cuando los pacientes presentan conductas agitadas o violentas, o bien depresiones graves con intentos autoliticos. Para que los Servicios de Pediatría pudieran hacerse cargo de estos casos se necesitaría un programa de interconsulta hospitalaria que llevara a cabo los tratamientos y el apoyo necesario, desarrollado por un equipo con for- mación especifica. Los programas de interconsulta hospitalaria no se realizan en muchos hospitales, y en los que se hacen, la mayoría están a cargo de los profesionales de adultos.

En cuanto a los recursos intermedios, hospitales de día, centros de día, hogares alternativos, etc., la situación es lamentable, tercermundista. Como todos sabemos son dispositivos imprescindibles para el tratamiento de casos graves en algunos casos alternativos y en otros complementarios a la hospitalización.

El hecho de que los trastornos graves (que precisan un ingreso) en niños y adolescentes no sean los más frecuentes (por otra parte como ocurre con los adultos, los mas frecuentes son los menos graves) no justifica de ninguna manera la escasez y precariedad de recursos y podemos asegurar que cuando se presentan, los programas ambulatorios son totalmente insuficientes.

Esperamos que el debate abierto sirva al menos para que las Administraciones Sanitarias tomen conciencia de la urgente necesidad de atender los trastornos en salud mental de niños y adolescentes, de forma adecuada y garantizando tanto la calidad asistencial como la continuidad de cuidados.

\footnotetext{
* Presidente AEN

**Vicepresidenta AEN
} 